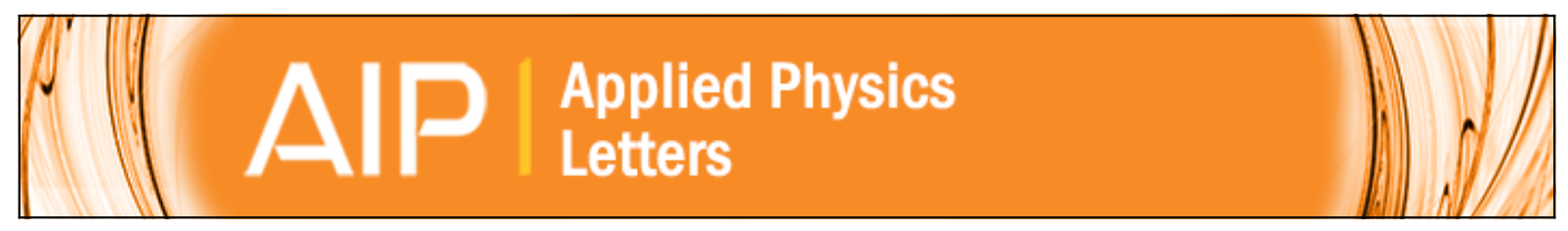

\title{
Polyheteroarylene films with intrinsic switching mechanism for nonvolatile memory applications
}

\author{
A. Zherebov, A. Lachinov, J. Genoe, and A. Tameev
}

Citation: Applied Physics Letters 92, 193302 (2008); doi: 10.1063/1.2912348

View online: http://dx.doi.org/10.1063/1.2912348

View Table of Contents: http://scitation.aip.org/content/aip/journal/apl/92/19?ver=pdfcov

Published by the AIP Publishing

\section{Articles you may be interested in}

Forming free resistive switching in graphene oxide thin film for thermally stable nonvolatile memory applications J. Appl. Phys. 114, 124508 (2013); 10.1063/1.4823734

Mechanism of resistive switching in $\mathrm{Cu} / \mathrm{AlOx} / \mathrm{W}$ nonvolatile memory structures J. Appl. Phys. 113, 164506 (2013); 10.1063/1.4803062

Polarization behavior of poly(vinylidene fluoride-trifluoroethylene) copolymer ferroelectric thin film capacitors for nonvolatile memory application in flexible electronics

J. Appl. Phys. 108, 094102 (2010); 10.1063/1.3500428

Bipolar switching characteristics of nonvolatile memory devices based on poly(3,4-

ethylenedioxythiophene):poly(styrenesulfonate) thin film

Appl. Phys. Lett. 93, 033309 (2008); 10.1063/1.2960998

Reproducible resistive switching in nonvolatile organic memories

Appl. Phys. Lett. 91, 192103 (2007); 10.1063/1.2806275

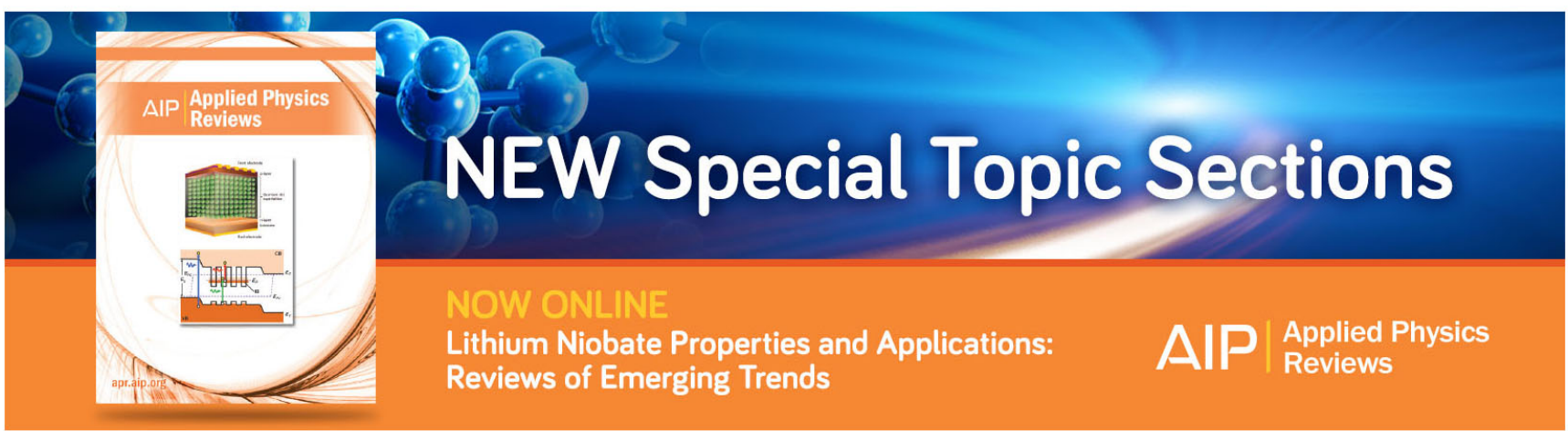




\title{
Polyheteroarylene films with intrinsic switching mechanism for nonvolatile memory applications
}

\author{
A. Zherebov, ${ }^{1, a)}$ A. Lachinov, ${ }^{1}$ J. Genoe, ${ }^{2}$ and A. Tameev ${ }^{3}$ \\ ${ }^{1}$ Institute of Physics of Molecules and Crystals of Ufa Scientific Centre of Russian Academy of Science, \\ Pr. Octyabrya 151, 450075 Ufa, Russia \\ ${ }^{2}$ IMEC, Kapeldreef 75, B-3001 Leuven, Belgium \\ ${ }^{3}$ A.N. Frumkin Institute of Physical Chemistry and Electrochemistry of Russian Academy of Science, \\ Leninsky Pr. 31, Bld. 4, 119991 Moscow, Russia
}

(Received 17 January 2008; accepted 31 March 2008; published online 12 May 2008)

\begin{abstract}
This paper presents a polymer material for the nonvolatile memory applications. Current-voltage characteristics show the reproducible nonvolatile switching effect. The effect is observed not only when using two metallic electrodes but also when using two semiconducting electrodes, which reveals the intrinsic nature of the switching phenomenon. (C) 2008 American Institute of Physics.

[DOI: 10.1063/1.2912348]
\end{abstract}

Switching effects in organic films have been known for about 40 years. $^{1}$ A lot of various organic materials such as pentacene, ${ }^{2}$ anthracene, ${ }^{3}$ copper tetracyanoquinodimethane complexes, ${ }^{4}$ polymethacrylate derivatives, ${ }^{5}$ and poly(2-methoxy-5(2' -ethyl)hexoxy-phenylenevinylene $)^{6}$ have been reported in this context. A number of interpretations have been proposed for the memory effect in organics including mechanisms involving charge transfer, ${ }^{4}$ charge trapping, ${ }^{7}$ electroreduction, ${ }^{8}$ etc. However, many authors, taking into consideration similarity of the switching effect in different materials, suppose it to be caused by oxidation of the electrodes ${ }^{9,10}$ or metallic filamentation originating from the metallic electrodes. ${ }^{10}$ In this case, the organic film plays the role of a passive matrix and does not influence the behavior of device much. This argument is very important for microelectronic industry because the possibility of scaling and adjusting of switching parameters is crucial for fabrication of commercial memory devices.

From this point of view, a polymer material with the intrinsic switching effect would be of great interest because its properties can be adjusted over a wide range by fabrication techniques and modifications of the molecule structure. Polyheteroarylenes are good candidates for this role. Switching induced by different external influences was reported in these materials. Among the switch inducing agents besides electric field ${ }^{11}$ are pressure, ${ }^{12}$ heating, ${ }^{13}$ change in the boundary conditions (e.g., a phase transition in one of the electrodes), ${ }^{14}$ and magnetic field. ${ }^{15}$ The existence of the different conductive states in these polymers is determined by the molecular properties. The polyheteroarylene molecules are known to possess two stable configurations. ${ }^{16}$ In the neutral state, the gap between the highest occupied molecular orbital and the lowest unoccupied molecular orbital is about $4.2 \mathrm{eV}$. In the charged state, a new deep electron level appears not far from the middle of the gap. Overlap of these states can lead to formation of a narrow band near the Fermi level which governs the polymer conductivity. In this state, the conductivity of the polymer can become comparable to

\footnotetext{
${ }^{a)}$ Present address: Laboratorium voor Vaste - Stoffysica en Magnetisme, K.U. Leuven, Celestijnenlaan 200D, B-3001 Leuven, Belgium. Electronic mail: Alexei.Zherebov@fys.kuleuven.be.
}

that of metals. Thus, manipulating the charge inside the polymer film, one can control its conductivity.

Together with unique electronic properties, polyheteroarylenes possess outstanding technological characteristics. They are soluble in many organic solvents and reveal excellent film forming properties, so that solid homogeneous films from $2 \mathrm{~nm}$ to $300 \mu \mathrm{m}$ thick can be prepared using dip, drop, spin coating, and printing techniques. The films are chemostable, thermostable (softening and destruction temperatures exceed $400{ }^{\circ} \mathrm{C}$ ), hard (modulus of elasticity $\sim 3000 \mathrm{MPa}$ ), and transparent (optical gap $\sim 4.2 \mathrm{eV}$ ). They can be operated in the air without any noticeable degradation. This work is the evaluation of this polymer as the nonvolatile memory material candidate.

Sandwich type metal/polymer/metal structures were used in the experiments. The bottom electrodes of metal (aluminum or gold) were deposited and lithographically patterned on the silicon with a thermally grown $100 \mathrm{~nm}$ silicon dioxide layer. The polymer films were spin coated from a $1 \%$ to $5 \%$ cyclohexanone solution at $1000-4500$ rpm which produced the films from 20 to $500 \mathrm{~nm}$ thick. Subsequently, the samples were annealed in low vacuum at $200{ }^{\circ} \mathrm{C}$ during $1 \mathrm{~h}$. Top electrodes of aluminum were thermally evaporated on the polymer film in vacuum through a shadow mask. All the electrodes were stripes of $100 \mathrm{~nm}$ thick and 50 to $200 \mu \mathrm{m}$ wide so that the active area was between 0.01 and $0.04 \mathrm{~mm}^{2}$.

A Keithley 2602 dual-channel system sourcemeter was used for the current-voltage $(I-V)$ characteristics measurements. A load resistor of $1-100 \mathrm{k} \Omega$ was connected to the measurement circuit to avoid overload of the sample. All the measurements were performed in the air at room temperature.

Typically, the initial resistance of the polymer film exceeds $10^{9} \Omega$. No switching was detected in the polymer films thicker than $400 \mathrm{~nm}$ using bias voltages of up to $40 \mathrm{~V}$. When the film gets thinner, typically monostable switching is observed.

However, when the polymer film thickness decreases below $100 \mathrm{~nm}$, reproducible switching between two conductive states (Fig. 1) is observed. The switching on voltage is typically in the range between -2 and $-3 \mathrm{~V}$ (the gold electrode is grounded), the switching off normally occurs at voltages 


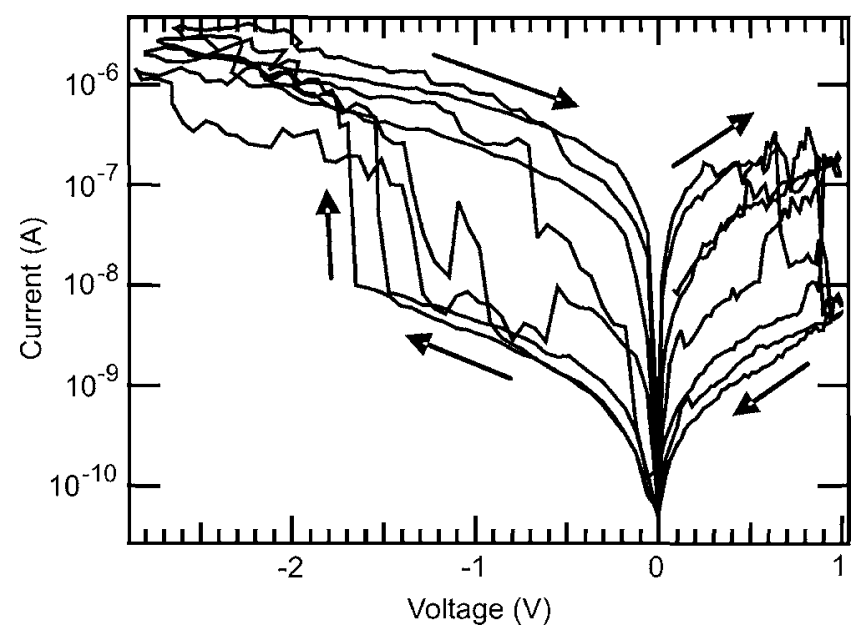

FIG. 1. I-V characteristics of the Au/polymer/Al sample. Polymer film thickness is $60 \mathrm{~nm}$. Au is grounded. Arrows indicate the way we sweep through the current-voltage characteristics.

between 1 and $2.5 \mathrm{~V}$, and the on/off current ratio is about 100.

In films less than $50 \mathrm{~nm}$ thick, switching on can occur at both polarities. Figure 2 shows the memory switching in the $20 \mathrm{~nm}$ thick polymer film. In contrast to the Fig. 1, switching on takes place in this case at positive bias on the aluminum electrode. The on/off current ratio in these films can reach 10000 . However, only a little number of switching cycles can be observed on such thin films.

More reproducible behavior is observed using the samples with both aluminum electrodes. Figure 3(a) shows 100 sequential cycles measured on the $\mathrm{Al} /$ polymer/Al sample. Figure 3(b) shows the on and off current in each cycle read at $2 \mathrm{~V}$. More than 3000 switching cycles were observed.

To exclude both the influence of metallic filamentation through the polymer film and switching in the aluminum oxide layer, and to confirm that the intrinsic polymer mechanism is responsible for the memory switching effect presented in this work, we performed similar experiments with indium tin oxide (ITO) electrodes. We used two glass plates with ITO stripes of 50-200 $\mu \mathrm{m}$ wide. On one of them, the polymer film was spin coated. The second glass plate was

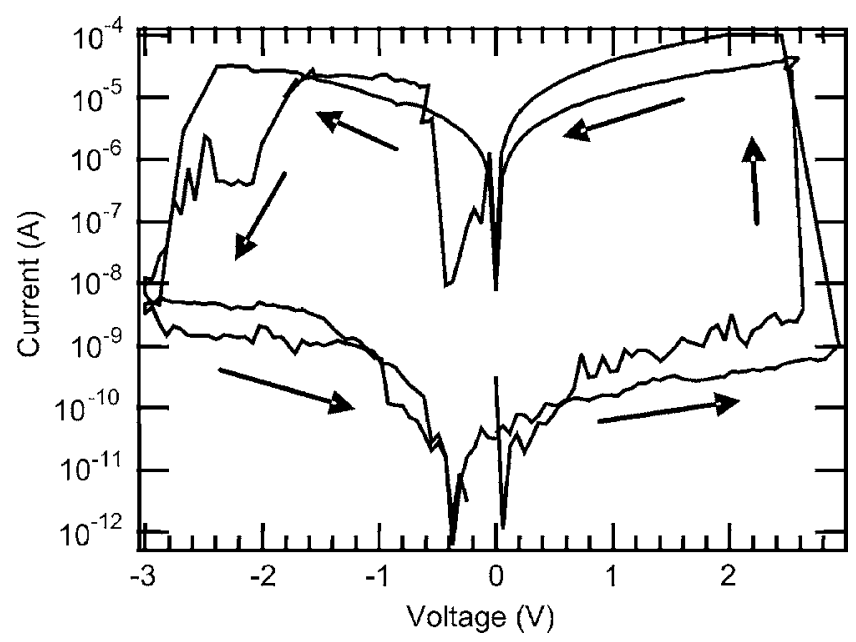

FIG. 2. I- $V$ characteristics of the Au/polymer/Al sample. Polymer film thickness is $20 \mathrm{~nm} \mathrm{Au}$ is grounded. Arrows indicate the way we sweep through the current-voltage characteristics.
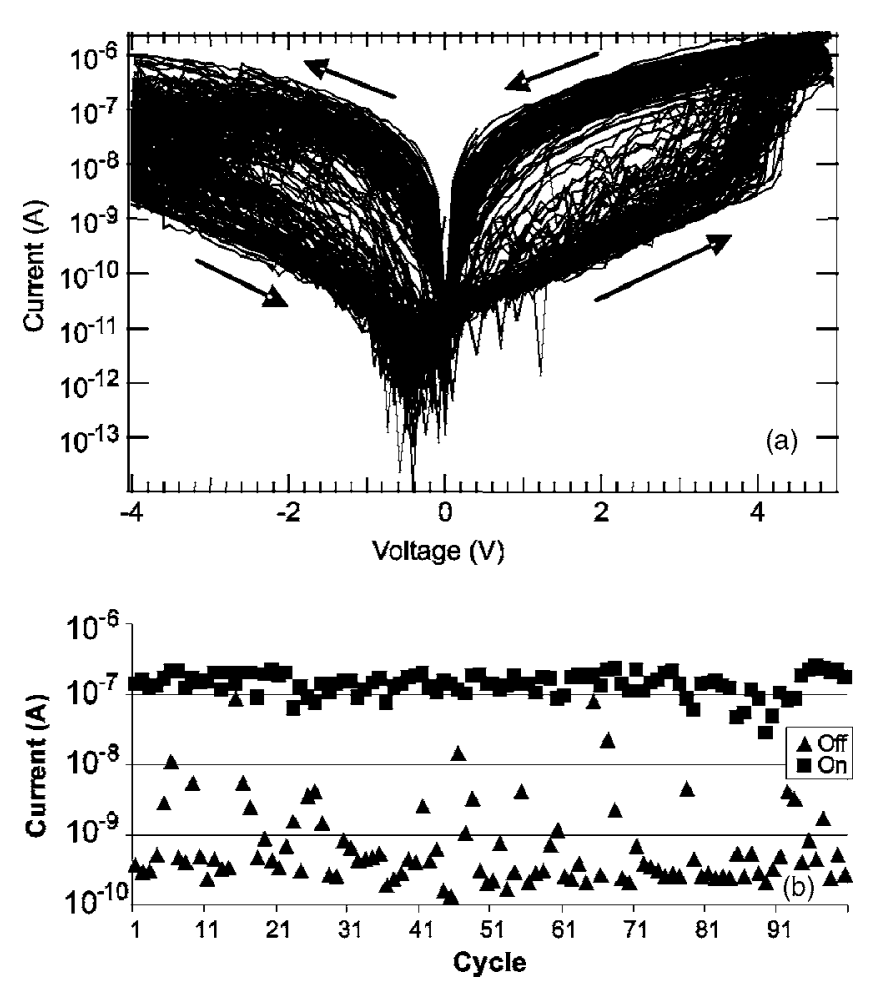

FIG. 3. (a) $I-V$ characteristics of the $\mathrm{Al} / \mathrm{polymer} / \mathrm{Al}$ sample. Polymer film thickness is $60 \mathrm{~nm}$. Arrows indicate the way we sweep through the currentvoltage characteristics. (b) On and off currents in each cycle read at $2 \mathrm{~V}$.

pressed to the top of the polymer sample. This yielded the same electrode configuration as in the above presented experiments, but without any metallic electrodes. ITO is generally considered as a degenerated semiconductor with reasonably high intrinsic conductivity. ${ }^{17}$ We know neither explicit reports on the switching effect in ITO nor on the metal filament grown from ITO electrodes in conditions similar to those described in this work.

The switching effect in the devices with ITO electrodes appears to be very similar to the effect with metallic electrodes (Fig. 4). However, the reproducibility was worse. This can be explained by lower reliability of pressed contacts as compared to the deposited ones. The existence and similarity of the characteristics of switching in the devices with ITO electrodes (that exclude metallic filamentation and oxide layer growth) to those observed with the metallic electrodes proved the intrinsic polymer mechanism of the effect.

In conclusion, in this work, we presented a polymer material for utilization in the nonvolatile memory devices.

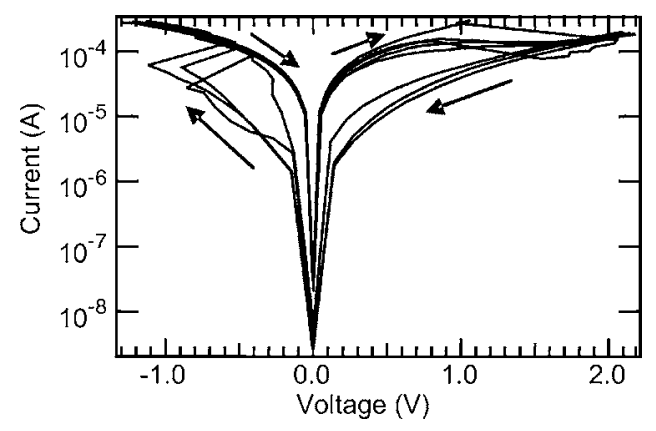

FIG. 4. $I-V$ characteristics of the ITO/polymer/ITO sample. Polymer film thickness is $60 \mathrm{~nm}$. Arrows indicate the way we sween through the currentvoltage characteristics. 
Films of this polymer reveal nonvolatile switching effects with the characteristics similar to those reported in other materials. The effect is based on the intrinsic molecular electronic mechanism and depends very little on the electrode material and dimension. The reproducibility is still not perfect. However, the intrinsic molecular electronic mechanism together with outstanding material stability, processability and possibility of structural modification make it promising for the nonvolatile memory applications.

A.Z., A.L., and A.T. acknowledge the BWTS-RFBR project No. 05-03-34827-MФ entitled "Electronic switching in thin films of organic semiconductors for advanced memory applications" for financial support.

\footnotetext{
${ }^{1}$ A. Szymanski, D. C. Larson, and M. M. Labes, Appl. Phys. Lett. 14, 88 (1969).

${ }^{2}$ D. Tondelier, K. Lmimouni, D. Vuillaume, C. Fery, and G. Haas, Appl. Phys. Lett. 85, 5763 (2004).

${ }^{3}$ A. R. Elsharkawi and K. C. Kao, J. Phys. Chem. Solids 38, 95 (1977).
}

${ }^{4}$ R. S. Potember, T. O. Poehler, and D. O. Cowan, Appl. Phys. Lett. 34, 405 (1979).

${ }^{5}$ D. M. Aguiar, J. A. Freire, and I. A. Hümmelgen, Adv. Mater. (Weinheim, Ger.) 12, 1063 (2000)

${ }^{6}$ M. Lauters, B. McCarthy, D. Sarid, and G. E. Jabbour, Appl. Phys. Lett. 89, 013507 (2006).

${ }^{7}$ J. Ouyang, C. W. Chu, C. R. Szmanda, L. Ma, and Y. Yang, Nat. Mater. 3, 918 (2004).

${ }^{8}$ A. Bandyopadhyay and A. J. Pal, Appl. Phys. Lett. 82, 1215 (2003).

${ }^{9}$ M. Colle, M. Buchel, and D. M. de Leeuw, Org. Electron. 7, 305 (2006).

${ }^{10}$ S. Karthauser, B. Lussem, M. Weides, M. Alba, A. Besmehn, R. Oligschlaeger, and R. Waser, J. Appl. Phys. 100, 094504 (2006).

${ }^{11}$ A. Yu. Zherebov and A. N. Lachinov, Synth. Met. 46, 181 (1992).

${ }^{12}$ A. Yu. Zherebov and A. N. Lachinov, Synth. Met. 44, 99 (1991).

${ }^{13}$ A. N. Lachinov, A. Yu. Zherebov, and M. G. Zolotukhin, Synth. Met. 59, 377 (1993).

${ }^{14}$ V. M. Kornilov and A. N. Lachinov, Synth. Met. 84, 893 (1997).

${ }^{15}$ A. N. Lachinov, N. V. Vorob'eva, and A. A. Lachinov, JETP Lett. 84, 604 (2006).

${ }^{16}$ N. Johansson, A. N. Lachinov, S. Stafstrom, and W. R. Salaneck, Synth. Met. 67, 319 (1994).

${ }^{17}$ J. C. C. Fan and J. B. Goodenough, J. Appl. Phys. 48, 3524 (1977). 